\title{
Activité parasitaire des Trichoderma vis-à-vis des champignons à sclérotes ; corrélation avec l'aptitude à la compétition dans un sol non sté- rile
}

\author{
Pierre DAVET \\ avec la collaboration techuique de Christine ROURE \\ I.N.R.A., Laboratoire de Biologie et Pathologie végétales de l'E.N.S.A., place Viala, Centre de Recherches de' \\ Montpellier, F 34060 Montpellier Cede.
}

Mots clés additionnels : Sclerotium rolfsii, Sclerotinia sclerotiorum, tri, compétition saprophytique.

The relative capacities of twenty clones of Trichoderma for destruction of Sclerotinia sclerotiorum (Lib) de Bary and Sclerotium rolfsii Sacc. sclerotia in a non sterile soil were almost similar. However, clone performances were different from those in a sterile soil of the same origin (ARTIGUES \& DAVET, 1984a), which showed that the soil microflora appreciably interferes with potential antagonistic abilities. On the other hand, the parasitic activity of the clones in a non-sterile soil varied as their competitive saprophytic ability, which had been measured in the same soil by an agar disk method (DAVFT \& CAMPOROTA, 1986). This measure of competitive saprophytic ability could therefore be used as a first criterion in screening for the most efficient clones.

Additional key words : Sclerotium rolfsii, Sclerotinia sclerotiorum, screening, saprophytic competition.

\section{INTRODUCTION}

La mise en évidence d'un comportement mycoparasitaire chez plusieurs espèces de Trichoderma Pers. a été suivie par de nombreux travaux de recherche qui permettent désormais d'envisager l'utilisation de ces propriétés pour une lutte biologique contre divers champignons phytopathogènes dans un avenir assez proche (PAPAvizAS, 1985). Cependant, tous les clones de Trichoderma n'ont pas les mêmes potentialités (ARTigues \& DAVET, 1984a). Aussi est-il indispensable de les trier et, dans cette perspective, il paraît nécessaire de s'interroger sur les critères qui permettraient un choix rapide des clones les plus efficaces vis-à-vis d'une cible donnée. Par exemple, en l'absence d'interférences avec la microflore du sol, la mesure des activités $\beta$ (1-3) glucanasique et chitinasique donne une assez bonne image des potentialités parasitaires des souches vis-à-vis des champignons à sclérotes (ARTigues \& DAVET, 1984b). Cependant, on peut se demander si ces critères sont encore suffisants pour identifier les clones les plus performants en situation de compétition, dans un sol non stérile. Les résultats présentés ci-dessous apporteront peut-être quelques éléments de réponse à cette question. 


\section{MATÉRIEL ET MÉTHODES}

\section{A. Champignons antagonistes}

Nous utilisons la collection de 20 isolements monospores de Trichoderma, décrite précédemment (ARTIGUES \& DAVET, 1984a). L'inoculum nécessaire aux essais est préparé dans des fioles de Roux (200 g de sable, $6 \mathrm{~g}$ de farine d'avoine, $30 \mathrm{ml}$ d'eau distillée; 2 autoclavages à $24 \mathrm{~h}$ d'intervalle). Les fioles sont placées dans une étuve à $28{ }^{\circ} \mathrm{C}$ durant 2 semaines; pendant cette période, elles sont secouées manuellement le $9^{\text {e }} \mathrm{j}$ pour améliorer la colonisation du milieu : elles sont maintenues 2 autres semaines à la température du laboratoire et à la lumière du jour. Leur contenu est alors séché à l'air, dans des conditions aseptiques, pendant $3 \mathrm{j}$. Après homogénéisation, un échantillon des poudres ainsi obtenues est prélevé pour déterminer leur teneur en propagules (DAVET, 1979). Le reste est stocké à $5{ }^{\circ} \mathrm{C}$ dans des boîtes étanches et constitue l'inoculum.

\section{B. Champignons cibles}

La souche de Sclerotium rolfsii Sacc. a été isolée sur poivron à Saint-Martin-de-Hinx (Landes). Le champignon est ensemencé sur milieu PDA en boîtes de Petri et cultivé à $24{ }^{\circ} \mathrm{C}$. Les sclérotes sont recueillis par grattage de la surface lorsque le milieu de culture est desséché, après 5 ou 6 semaines d'incubation.

Les sclérotes de Sclerotinia sclerotiorum (Lib.) De Bary proviennent d'un inoculum naturel, recueilli après le battage d'une parcelle expérimentale de colza du Centre Technique Interprofessionnel des Oléagineux Métropolitains (CETIOM), située à Saint Pathus (Seine-et-Marne). Ils hébergent une mycoflore naturelle comprenant moins de 1 p. 100 de Trichoderma. Ils sont conservés au sec, à la température du laboratoire.

\section{Sol}

La terre provient d'un jardin de Montpellier. C'est un sol sablo-limoneux de $\mathrm{pH}\left(\mathrm{Ca} \mathrm{Cl}_{2}\right)=6,7$, contenant 1,94 p. 100 de carbone organique. La quantité nécessaire aux essais est tamisée, puis stockée au laboratoire dans un sac en polyéthylène fermé.

\section{Confrontations entre les sclérotes et les antagonis- tes}

Dans les essais comprenant toute la collection, on mélange, à $200 \mathrm{ml}$ de terre, un inoculum constitué de $10 \mathrm{ml}$ de sable ensemencé séché. Dans d'autres essais, réalisés avec des échantillons réduits de 12 clones, l'inoculum est ajusté de façon à avoir exactement une proportion de $10^{5}$ propagules par $\mathrm{g}$ de terre. Cette concentration représente, pour la plupart des clones, un volume analogue au précédent; pour certains cependant, le volume nécessaire est nettement différent (de 2 à $60 \mathrm{ml}$ ). On ajoute à ce mélange $150 \mathrm{mg}$ de sclérotes de $S$. rolfsii ou bien 170 sclérotes de $S$. scle- rotiorum (le poids des sclérotes de ce champignon étant très variable, on ne peut procéder par pesée). L'ensemble est homogénéisé, humidifié à 70 p. 100 de la capacité de rétention et introduit dans un sac de polyéthylène scellé d'un ruban adhésif. Les sacs sont maintenus à 22 ou à $28^{\circ} \mathrm{C}$ pendant 3 semaines.

\section{E. Estimation de l'activité parasitaire}

Le contenu des sacs est tamisé sous un courant d'eau. On recueille 100 sclérotes de $S$. rolfsii par sac (tamis à mailles de $250 \mu \mathrm{m}$ ) et 150 sclérotes de $S$. sclerotiorum (mailles de $500 \mu \mathrm{m}$ ). Lorsqu'ils sont mous, les sclérotes sont considérés comme détruits et comptabilisés d'emblée, car des essais préliminaires nous ont montré qu'ils étaient, dans ce cas, complètement parasités et incapables de germer. Les autres sclérotes sont mis en culture sur milieu PDA acidifié $(100 \mathrm{mg} / \mathrm{l}$ d'acide citrique), après désinfection superficielle à l'hypochlorite de sodium à 5 p. 100 pendant $3 \mathrm{mn}$. Les cultures sont observées après $5 \mathrm{j}$ d'incubation à l'étuve à $28^{\circ} \mathrm{C}$ et $2 \mathrm{j}$ à la lumière du jour, à la température du laboratoire. Seuls sont considérés comme attaqués les sclérotes qui fournissent exclusivement une colonie de Trichoderma sp. Les sclérotes qui sont colonisés mais parviennent à germer ne sont pas pris en compte. Chaque essai est répété au moins 3 fois.

\section{F. Notations}

On calcule pour chaque clone un « taux de destruction des sclérotes $»=\frac{\mathrm{n} 1+\mathrm{n} 2}{\mathrm{~N}} \times 100$, où $\mathrm{n} 1$ est le nombre de sclérotes mous, $\mathrm{n} 2$ le nombre de colonies pures de Trichoderma sp. et $\mathrm{N}$ le nombre total de sclérotes comptés. Les analyses de variance sont faites après transformation des pourcentages en arc sinus et les moyennes sont comparées par la méthode de NEWMAN \& KEULS. Les corrélations entre classements sont calculées par la méthode de SPEARMAN.

\section{RÉSULTATS}

\section{A. Comparaison du parasitisme vis-à-vis des deux types de sclérotes}

Il existe des différences importantes dans les aptitudes parasitaires des clones vis-à-vis des 2 champignons cibles. La variabilité des résultats d'une répétition à une autre est moins grande avec $S$. sclerotiorum, sans doute parce que la totalité des essais est réalisée avec un seul et même lot de sclérotes de ce champignon. Cependant, les clones qui se montrent efficaces contre S. sclerotiorum $(\mathrm{HH} 3, \mathrm{~B} 1,24, \mathrm{MB})$ sont également efficaces vis-à-vis de $S$. rolfsii et les clones médiocres dans les confrontations avec l'un de ces parasites sont aussi médiocres avec l'autre (tabl. 1). La corrélation entre les 2 classements est significative au seuil de 1 p. $100(\mathrm{r}=0,70)$. 
TABLEAU 1

Activité parasitaire des clones de Trichoderma vis-à-vis de Sclerotinia sclerotiorum et de Sclerotium rolfsii, à $28{ }^{\circ} \mathrm{C}$. Parasitic activity of some Trichoderma clones against S. sclerotiorum and S. rolfsii, at $28{ }^{\circ} \mathrm{C}$.

\begin{tabular}{|c|c|c|c|c|}
\hline \multirow{2}{*}{$\begin{array}{c}\text { Clones } \\
\text { antagonistes }\end{array}$} & \multicolumn{2}{|c|}{$\begin{array}{l}\text { Taux de destruction des sclérotes } \\
\text { (en p. 100) }\end{array}$} & \multirow{2}{*}{$\begin{array}{l}\text { Classement selon } \\
\text { la compétitivité }\end{array}$} & \multirow{2}{*}{$\begin{array}{c}\text { Classement selon } \\
\text { l'activité } \\
\text { enzymatique }\end{array}$} \\
\hline & S. sclerotiorum & S. rolfsii & & \\
\hline HH 3 & 90,2 a & $33,3 \mathrm{ab}$ & 5 & 6 \\
\hline B 1 & 84,9 ab & 54,3 a & 2 & 18 \\
\hline 3160 & $72,7 \mathrm{abc}$ & $5,5 \mathrm{ab}$ & 3 & 14 \\
\hline 24 & 67,6 abcd & $31,8 \mathrm{ab}$ & 1 & 16 \\
\hline LX 1 & 64,9 abcde & $29,5 \mathrm{ab}$ & 12 & 12 \\
\hline $\mathrm{HH} 1$ & 64,2 abcde & $31,0 \mathrm{ab}$ & 4 & 1 \\
\hline $\mathrm{MB}$ & 56,0 abcdef & $35,8 \mathrm{ab}$ & 6 & 9 \\
\hline B 140 & 55,8 abcdef & $24,0 \mathrm{ab}$ & 13 & 8 \\
\hline LW 1 & 45,3 bcdefg & $3,0 \mathrm{~b}$ & 16 & 7 \\
\hline LR 1 & 42,2 cdefg & $1,0 \mathrm{~b}$ & 15 & 3 \\
\hline $\mathrm{KZ} 1$ & 38,9 cdefg & $18,0 \mathrm{ab}$ & 11 & 15 \\
\hline LN 3 & 38,9 cdefg & $5,8 \mathrm{ab}$ & 18 & 11 \\
\hline LN 1 & 36,5 cdefg & $0,5 \mathrm{~b}$ & 19 & 2 \\
\hline V 1 & 32,9 cdefg & $19,3 \mathrm{ab}$ & 7 & 10 \\
\hline LR 3 & 28,5 cdefg & $3,3 \mathrm{~b}$ & 9 & 4 \\
\hline LK 1 & 20,2 defgh & $24,0 \mathrm{ab}$ & 8 & 13 \\
\hline JE 1 & $17,1 \mathrm{efgl}$ & $7,5 \mathrm{ab}$ & 10 & 5 \\
\hline LX 3 & $13,1 \mathrm{fgh}$ & $5,0 \mathrm{~b}$ & 14 & 19 \\
\hline MD 1 & $8,7 \mathrm{gh}$ & $0,5 \mathrm{~b}$ & 17 & 17 \\
\hline JI 1 & $0,7 \mathrm{~h}$ & $0,3 b$ & 20 & 20 \\
\hline
\end{tabular}

Le volume d'inoculum introduit dans le sol est de 5 p. 100 pour tous les clones. Les valeurs suivies d'une même lettre ne sont pas significativement différentes au seuil de 5 p. 100 (test de NEwMAN \& KevLs). On a rappelé dans ce tableau le classement des clones selon leur compétitivité, établi par la méthode des pastilles gélosées (DAvET \& CAMPorotA, 1986) dans des conditions d'expérimentation comparables, et le classement selon les activités $\beta(1-3)$ glucanasique et chitinasique (ARTIGUES \& DAVET, 1984b).

The volume of inoculum used was, for each clone, $5 \mathrm{~g}$ per $100 \mathrm{~g}$ of soil. Figures followed by the same letter were not significantiy difterent at 5 per 100 level, according to NEWMAN \& KEULS' test. Classifications of clones according to their competitive saprophytic ability (agar disk method : DAVET \& CAMPOROTA, 1986) and according to their $\beta(1-3)$ glucanase and chitinase activities (ARTIGUES \& DAVET, 1984 $b$ ) have also been recorded in this table.

\section{B. Effet du mode d'apport de l'inoculum}

Si l'on compare les résultats des essais où la quantité de propagules utilisée reste constante pour tous les clones (tabl. 2) aux données obtenues avec un volume d'inoculum constant (tabl. 1), on trouve une corrélation de 0,54 (significative à 7 p. 100) entre les classements des clones. Des variations, même importantes, dans les concentrations en propagules (essais à volume d'inoculum constant) n'empêchent donc pas les différences entre les souches de se manifester.

\section{Comparaison entre le pouvoir parasitaire, I'apti- tude à la compétition et certaines activités enzyma- tiques}

Les classements des clones en fonction de leur aptitude à la compétition sont rappelés dans les tableaux 1 et 2 . Les résultats auxquels nous nous référons et la méthode par laquelle nous les avons obtenus ont été exposés récemment (DAVET \& CAMPOROTA, 1986). Il est ainsi possible de comparer les places obtenues par les différentes souches selon que l'on note leur activité parasitaire ou leur compétitivité, dans des conditions analogues d'expérimentation : il existe une très bonne corrélation entre ces classements dans toutes les séries étudiées (tabl. 3) : le coefficient de corrélation est compris entre 0,65 et 0,78 . Par contre, il n'y a pas de corrélation nette entre le pouvoir parasitaire en terre non stérile et les activités $\beta$ (1-3) glucanasique et chitinasique, telles qu'elles ont été évaluées dans un travail antérieur (ARTIGUES \& DAVET, 1984b).

\section{DISCUSSION}

Les sclérotes de $S$. sclerotiorum sont plus fortement attaqués que ceux de $S$. rolfsii. Nous avions déjà constaté, en terre stérile, que les sclérotes de $S$. minor et de $S$. sclerotiorum étaient plus intensément détruits que ceux de $S$. rolfsii (ARTIGUES \& DAVET, 1984a). Ici, la fragilité des sclérotes de $S$. sclerotiorum est due sans doute à la fois à des caractères propres à l'espèce et au fait qu'ils ont été formés dans des conditions naturelles. Selon MERRIMAN (1976), en effet, les sclérotes produits in vitro ont un cortex plus épais et plus régulier, assurant une protection plus efficace.

Les classements des clones ne sont plus les mêmes selon que le sol dans lequel on opère a été stérilisé ou non : ceci montre bien la nécessité de prendre en compte l'aptitude des Trichoderma à la compétition. Cependant, dans un cas comme dans l'autre, les clones les plus actifs vis-à-vis de $S$. sclerotiorum sont aussi les plus efficaces vis-à-vis de $S$. rolfsiii. S. rolfsii, qui se développe rapidement et produit en grand nombre des sclérotes faciles à manipuler, constitue un modèle intéressant. Selon HENIS et al. (1984), le clas- 
TABLEAU 2

Activité parasituire des clones de Trichoderma vis-ù-vis de $\mathrm{S}$. rolfsii, estimée dans des essais où le taux de propagules est, dans tous les cas, égal à $10^{5}$ par $g$ de terre.

Parasitic activity of some Trichoderma clones against $\mathrm{S}$. rolfsii. In these trials, propagule numbers were, in every case, $10^{5}$ per $g$ of soil.

\begin{tabular}{|c|c|c|c|c|}
\hline \multirow{2}{*}{$\begin{array}{l}\text { Clones } \\
\text { antagonistes }\end{array}$} & \multicolumn{2}{|c|}{ Incubation à $22{ }^{\circ} \mathrm{C}$} & \multicolumn{2}{|c|}{ Incubation à $28^{\circ} \mathrm{C}$} \\
\hline & $\begin{array}{c}\text { Taux de } \\
\text { destruction }\end{array}$ & $\begin{array}{l}\text { Classement selon } \\
\text { la competitivité }\end{array}$ & $\begin{array}{c}\text { Taux de } \\
\text { destruction }\end{array}$ & $\begin{array}{l}\text { Classement selon } \\
\text { la compétitivité }\end{array}$ \\
\hline B 1 & 26,7 & 1 & 45,0 & 2 \\
\hline B 140 & 25,3 & 8 & 39,5 & 8 \\
\hline 24 & 20,0 & 2 & 28,5 & 4 \\
\hline V 1 & 16,7 & 6 & 34,0 & 5 \\
\hline KZ 1 & 15,7 & 5 & 23,5 & 7 \\
\hline $\mathrm{HH} 1$ & 14,7 & 7 & 30,0 & 3 \\
\hline HH 3 & 12,0 & 4 & 52,0 & 1 \\
\hline $\mathrm{MB}$ & 10,3 & 3 & 10,5 & 6 \\
\hline LW 1 & 9,3 & 10 & 27,0 & 12 \\
\hline LX 3 & 6,0 & 9 & 7,0 & 9 \\
\hline LR 3 & 4,3 & 12 & 30,0 & 10 \\
\hline MD 1 & 1,7 & 11 & 10,5 & 11 \\
\hline
\end{tabular}

On a indiqué également le classement des clones selon leur competitivité, établi précédemment dans les mêmes conditions d'expérimentation (DAvET \& CAMPOROTA, 1986).

Competitive saprophytic ability had already been studied, in the same experimental conditions (DAVET \& CAMPOROTA, 1986), and the resulting classifications are given in the table.

TABLEAU 3

Corrélations entre les classements des clones de Trichoderma selon leur activité parasitaire vis-à-vis de $\mathrm{S}$. rolfsii ou de $\mathrm{S}$. sclerotiorum, et selon leur aptitude à la compétition, mesurée par la méthode des pastilles gélosées. Les corrélations sont significatives à l p. lo0 (**) ou à 5 p. $100\left(^{*}\right)$.

Correlations hetween the ranking of Trichoderma clones when they were classified according to their ability to destroy $\mathrm{S}$. rolfsii or $\mathrm{S}$. sclerotiorum scterotia, and according to their saprophytic competitive ability, evaluated by the agar disk method. The correlations were significant at $1 \%(* *)$ or at $5 \%(*)$ level.

\begin{tabular}{|c|c|c|c|c|}
\hline \multirow[b]{2}{*}{$\begin{array}{l}\text { Condition } \\
\text { des } \\
\text { essais }\end{array}$} & \multirow{2}{*}{$\begin{array}{c}\text { Incubation à } 22{ }^{\circ} \mathrm{C} \\
S \text {. rolfsii } \\
\text { même taux de } \\
\text { propagules pour } \\
\text { tous les clones }\end{array}$} & \multicolumn{3}{|c|}{ Incubation à $28{ }^{\circ} \mathrm{C}$} \\
\hline & & $\begin{array}{l}\text { même taux de } \\
\text { propagules pour } \\
\text { tous les clones }\end{array}$ & $\begin{array}{l}\text { même volume } \\
\text { d'inoculum pour } \\
\text { tous les clones }\end{array}$ & $\begin{array}{l}\text { S. sclerotiorum } \\
\text { même volume } \\
\text { d'inoculum pour } \\
\text { tous les clones }\end{array}$ \\
\hline Corrélations & $0,72 * *$ & $0,65^{*}$ & $0,78 * *$ & $0,65 *$ \\
\hline
\end{tabular}

sement des Trichoderma serait indépendant de la souche de $S$. rolfsii utilisée dans les confrontations. La variabilité des résultats pourrait être réduite en amenant tous les lots de sclérotes, avant leur emploi, à un état de dessication équivalent. Henis \& Papavizas (1983) utilisent comme agent desséchant le sulfate de calcium, dans une enceinte à $20^{\circ} \mathrm{C}$.

Les différences observées entre les clones illustrent la variabilité des populations naturelles et confirment bien la nécessité d'un tri. Il est intéressant de constater que les classements demeurent sensiblement les mêmes de 22 à $28{ }^{\circ} \mathrm{C}$ et que les comparaisons entre les clones n'exigent pas un ajustement trop rigoureux des concentrations d'inoculum, ce qui simplifie les conditions d'expérimentation. Mais les confrontations entre sclérotes et antagonistes sont longues, fastidieuses, et la dispersion des résultats oblige à réaliser plusieurs répétitions. D'autre part, la mesure de quelques activités enzymạtiques ( $\beta(1-3)$ glucanase, chitinase), prises comme reflets de l'activité mycoparasitaire, ne semble pas rendre compte correctement, à elle seule, du comportement des clones dans un sol non stérile ; des critères d'activité saprophytique semblent également nécessaires. Il n'est cependant pas souhaitable, sur un plan pratique, de multiplier le nombre de paramètres. Aussi est-il intéressant de constater qu'il existe une bonne corrélation entre l'activité antagoniste et l'aptitude globale à la compétition. L'aptitude à la compétition, facile à évaluer par la méthode des pastilles gélosées (DAvET \& CAMPOROTA, 1986), pourrait constituer un bon critère pour une première étape de sélection des clones les plus intéressants dans la pratique. Selon les travaux actuellement en cours dans notre laboratoire, le classement des clones ne semble pas modifié par la nature du sol utilisé dans les essais. 


\section{RÉFÉRENCES BIBLIOGRAPHIQUES}

Artigues M., Davet P., 1984a. Comparaison des aptitudes parasitaires de clones de Trichoderma vis-à-vis de quelques champignons à sclérotes. Soil Biol. Biochem., 16, 413-417.

Artigues M., Davet P., 1984b. Activités $\beta$ (1-3) glucanasique et clitinasique de quelques champignons, en relation avec leur aptitude à détruire les sclérotes de Corticium rolfsii dans de la terre stérile. Soil Biol. Biochem., 16, 527-528.

Davet P.. 1979. Technique pour l'analyse des populations de Trichoderma et de Gliocladium virens dans le sol. Ann. Phytopathol., 11, 529-533.

Davet P., Camporota P.. 1986. Etude comparative de quelques methodes d'estimation de l'aptitude à la compétition saprophytique dans le sol des Trichoderma. Agronomie, 6 (6), 575-581.
Henis Y., Papavizas G. C.. 1983. Factors affecting germinability and susceptibility to attack of sclerotia of Sclerotium rolfsii by Trichoderma harzianum in field soils. Phytopathology, 73, 1469-1474.

Henis Y., Lewis J. A., Papavizas G. C., 1984. Interactions between Sclerotium rolfsii and Trichoderma spp.: relationship between antagonism and disease control. Soil Biol. Biochem., 16, 391-395.

Merriman P. R.. 1976. Survival of sclerotia of Sclerotinia sclerotiorum in soil. Soil Biol. Biochem., 8, 385-389.

Papavizas G. C., 1985. Trichoderma and Gliocladium : biology, ecology, and potential for biocontrol. Annu. Rev. Phytopathol., 23. 23-54. 